\title{
ON FIXED POINTS OF GENERALIZED SET-VALUED CONTRACTIONS
}

\author{
S. BENAHMED and D. AZÉ
}

(Received 28 November 2008)

\begin{abstract}
Using a variational method introduced in [D. Azé and J.-N. Corvellec, 'A variational method in fixed point results with inwardness conditions', Proc. Amer. Math. Soc. 134(12) (2006), 3577-3583], deriving directly from the Ekeland principle, we give a general result on the existence of a fixed point for a very general class of multifunctions, generalizing the recent results of [Y. Feng and S. Liu, 'Fixed point theorems for multi-valued contractive mappings and multi-valued Caristi type mappings', J. Math. Anal. Appl. 317(1) (2006), 103-112; D. Klim and D. Wardowski, 'Fixed point theorems for set-valued contractions in complete metric spaces', J. Math. Anal. Appl. 334(1) (2007), 132-139]. Moreover, we give a sharp estimate for the distance to the fixed-points set.
\end{abstract}

2000 Mathematics subject classification: primary 54H25, 47H10.

Keywords and phrases: complete metric spaces, generalized set-valued contractions, Ekeland's variational principal.

\section{Introduction}

Recently some interesting extensions of Nadler's theorem (see [10]) were given in $[6,8]$; this was the first generalization to multifunctions of the classical BanachPicard theorem. In the papers quoted, the authors observed that the assumption that the multifunction is a contraction with respect to the Hausdorff metric could be slightly weakened by requiring only local information on the approximate projection of a point to its image. This observation was anticipated in [1, Example 1.6]. In this work we give a general result on the existence of a fixed point for a large class of multifunctions satisfying a very weak contraction assumption. Moreover, a sharp estimate for the distance to the fixed-points set is given. As a by-product, we derive a very light version of the Banach-Picard theorem.

\section{A basic lemma}

Definition 2.1. Let $(X, d)$ be a metric space, and let $f: X \rightarrow \mathbb{R} \cup\{+\infty\}$ be a function. A point $x \in X$ is said to be a $d$-point of $f$ if

$$
f(x)<f(z)+d(z, x) \quad \forall z \in X \backslash\{x\} .
$$

(C) 2009 Australian Mathematical Publishing Association Inc. 0004-9727/2009 \$16.00 
Here is the well-known Ekeland variational principle in its simpler form (see $[4,5,11,15])$.

\section{THEOREM 2.2. The following are equivalent:}

(a) the metric space $(X, d)$ is complete;

(b) every proper (not identically equal to $+\infty$ ), lower semicontinuous, and lowerbounded function $f: X \rightarrow \mathbb{R} \cup\{+\infty\}$ has a d-point.

Given $x \in X$, let us set $M(x)=\{z \in X \mid f(z)+d(x, z) \leq f(x)\}$. It is an immediate consequence of the triangle inequality that a $d$-point of the restriction of $f$ to the subset $M(x)$ is a $d$-point of $f$ on the whole of $X$. Thus we have the following result.

Corollary 2.3. Let $(X, d)$ be a complete metric space. Assume that the function $f: X \rightarrow \mathbb{R} \cup\{+\infty\}$ is proper lower semicontinuous and bounded from below. Then, for all $x \in X$, there exists a d-point of $f$ belonging to $M(x)$.

For $\lambda \in \mathbb{R}$, we denote by $[f \leq \lambda]$ the sublevel set $f^{-1}((-\infty, \lambda])$ and we define $[f<\lambda]$, $[f>\lambda]$, and similar notation analogously. The following simple lemma along the lines of $[2,7,9,14]$ is our basic tool in what follows.

LEMMA 2.4. Let $f: X \rightarrow[0,+\infty]$ be a proper lower semicontinuous function defined on a complete metric space $(X, d)$, and let $0<\mu \leq+\infty$ be such that $[f<\mu] \neq \emptyset$. Assume that

$$
\forall x \in[0<f<\mu] \exists z \neq x \text { such that } f(z)+d(x, z) \leq f(x) .
$$

Then $[f \leq 0] \neq \emptyset$, and, for all $x \in[f<\mu]$, we can find $z \in[f \leq 0]$ such that $d(x, z) \leq$ $f(x)$.

Proof. Given $x \in[f<\mu]$, then $M(x) \subset[f<\mu]$. Then, a $d$-point $z$ of $f$ which belongs to $M(x)$, whose existence is guaranteed by Corollary 2.3 , is in $[f \leq 0]$ since, from our assumption, an element of $[0<f<\mu]$ is not a $d$-point, and $z$ satisfies

$$
d(x, z) \leq f(z)+d(x, z) \leq f(x) .
$$

\section{Generalized contractions}

A multifunction $T$ from a set $X$ into a set $Y$ is a subset $T \subset X \times Y$. For any $x \in X$, we define $T(x)=\{y \in Y \mid(x, y) \in T\}$. The domain of the multifunction $T$ is the set of those $x \in X$ such that $T(x) \neq \emptyset$. We shall always assume that the domain of $T$ is nonempty. A fixed point of a multifunction $T \subset X \times X$ is a point $x \in X$ such that $x \in T(x)$. We shall denote by $\mathcal{F}_{T}$ the set of fixed points of $T$. Given a subset $C \subset X$ of a metric space and given $x \in X$, we set $d(x, C)=\inf _{z \in C} d(x, z)$ with the convention $d(x, C)=+\infty$ if $C=\emptyset$. For $C, D \subset X$, we shall also use $e(C, D)=\sup _{x \in C} d(x, D)$ with the conventions $e(C, D)=0$ if $C=\emptyset$ and $e(C, \emptyset)=+\infty$ if $C \neq \emptyset$. As is well known, $e(C, D)=\sup _{z \in X}(d(z, D)-d(z, C))$. 
Given functions $\kappa:(0,+\infty) \rightarrow[0,1)$ and $b:(0,+\infty) \rightarrow[\underline{b}, 1]$, where $\underline{b} \in(0,1]$, and given a multifunction $T \subset D \times X$ defined on a subset $D$ of a metric space $X$, we will say that $T$ satisfies assumption $(\mathcal{H})$ if, setting $d_{x}=d(x, T(x))$, we have $(\mathcal{H})\left\{\begin{array}{l}\text { for all } x \in D \text { such that } d(x, T(x))>0, \text { there exists } z \in D \backslash\{x\} \text { such that } \\ b\left(d_{x}\right) d(x, z) \leq d(x, T(x)) \leq d(x, z) \text { and } d(z, T(z)) \leq \kappa(d(x, z)) d(x, z) .\end{array}\right.$

It is natural to assume that the function $b(\cdot)$ is nonincreasing since we need less information when $d(x, T(x))$ decreases. Assuming that $D=X$, it is clear that if $T \subset X \times X$ is a multifunction such that $e(T(x), T(z)) \leq \kappa(d(x, z)) d(x, z)$ for all $x, z \in X$, an assumption used for example in [12, 14], then assumption $(\mathcal{H})$ is in force (taking $z \in T(x)$ such that $b\left(d_{x}\right) d(x, z) \leq d(x, T(x))$, and $d(z, T(z)) \leq$ $e(T(x), T(z)) \leq \kappa(d(x, z)) d(x, z))$.

EXAMPLE 3.1. Observe that the setting developed by Klim and Wardowski in [8] is contained in our framework. Namely, if $T \subset X \times X$ is a multifunction and if $b$ : $(0,+\infty) \longrightarrow(0,1)$ is a function, let us define, for all $x \in X$ such that $d(x, T(x))>0$, the set

$$
I_{b}^{x}=\left\{z \in T(x): b\left(d_{x}\right) d(x, z) \leq d(x, T(x))\right\},
$$

so that $I_{b}^{x}$ is nonempty. A strengthened version of assumption $(\mathcal{H})$ is then

$$
\begin{aligned}
& \forall x \in X \text { such that } d(x, T(x))>0, \exists z \in I_{b}^{x} \\
& \text { such that } d(z, T(z)) \leq \kappa(d(x, z)) d(x, z) .
\end{aligned}
$$

In the case where $b(t) \equiv b \in(0,1)$, we recover the setting of [8]. Observe also that our framework allows non-self multifunctions, that is, multifunctions defined on a subset $D$ of $X$ with values in $X$. We stress the fact that the point $z$ in assumption $(\mathcal{H})$ is not required to belong to $T(x)$, in such a way that there is no Lipschitz property for $T$ in this definition.

The following lemma is a significant extension of a result of Semenov in [13].

LEMMA 3.2. Let $(X, d)$ be a metric space and let $T \subset D \times X$ be a multifunction defined on a subset $D$ of $X$. Assume that there exist a function $\kappa:(0,+\infty) \rightarrow[0,1)$ and a nonincreasing function $b:(0,+\infty) \rightarrow[\underline{b}, 1]$ where $\underline{b} \in(0,1]$, such that $\kappa(\cdot)<$ $b(\cdot)$ and:

(1) T satisfies assumption $(\mathcal{H})$;

(2) $\limsup _{t \downarrow s} b(t)^{-1} \kappa(t)<1$ for all $s>0$.

Then $\inf _{x \in D} d(x, T(x))=0$.

Proof. Let $x_{0} \in D$. We may assume that $d\left(x_{0}, T\left(x_{0}\right)\right)>0$ (otherwise there is nothing to prove). Assume that there are known $x_{0}, \ldots, x_{n} \in D$ such that, for all $k \in\{0, \ldots, n\}, d\left(x_{k}, T\left(x_{k}\right)\right)>0$ and for all $k \in\{0, \ldots, n-1\}$,

$$
\left\{\begin{array}{l}
b\left(d_{x_{k}}\right) d\left(x_{k}, x_{k+1}\right) \leq d\left(x_{k}, T\left(x_{k}\right)\right) \leq d\left(x_{k}, x_{k+1}\right), \\
d\left(x_{k+1}, T\left(x_{k+1}\right)\right) \leq \kappa\left(d\left(x_{k}, x_{k+1}\right)\right) d\left(x_{k}, x_{k+1}\right)
\end{array}\right.
$$


From our assumptions, we can find a point $x_{n+1} \in D$ such that

$$
\left\{\begin{array}{l}
b\left(d_{x_{n}}\right) d\left(x_{n}, x_{n+1}\right) \leq d\left(x_{n}, T\left(x_{n}\right)\right) \leq d\left(x_{n}, x_{n+1}\right), \\
d\left(x_{n+1}, T\left(x_{n+1}\right)\right) \leq \kappa\left(d\left(x_{n}, x_{n+1}\right)\right) d\left(x_{n}, x_{n+1}\right) .
\end{array}\right.
$$

If $x_{n+1}=x_{n}$, then $d\left(x_{n+1}, T\left(x_{n+1}\right)\right)=0$, so that $\inf _{x \in D} d(x, T(x))=0$. If $x_{n+1} \neq$ $x_{n}$, we derive from (3.2), using the facts that $b(\cdot)$ is nonincreasing and $\kappa(\cdot)<b(\cdot)$, that

$$
\begin{aligned}
d\left(x_{n+1}, T\left(x_{n+1}\right)\right) & \leq \frac{\kappa\left(d\left(x_{n}, x_{n+1}\right)\right)}{b\left(d_{x_{n}}\right)} b\left(d_{x_{n}}\right) d\left(x_{n}, x_{n+1}\right) \\
& \leq c\left(d\left(x_{n}, x_{n+1}\right)\right) d\left(x_{n}, T\left(x_{n}\right)\right)
\end{aligned}
$$

where $c(t)=b(t)^{-1} \kappa(t)$. In particular, $d\left(x_{n+1}, T\left(x_{n+1}\right)\right) \leq d\left(x_{n}, T\left(x_{n}\right)\right)$. Moreover, by (3.1) and using again the fact that $\kappa(\cdot)<b(\cdot)$, we have

$$
d_{x_{n}}=d\left(x_{n}, T\left(x_{n}\right)\right) \leq b\left(d\left(x_{n-1}, x_{n}\right)\right) d\left(x_{n-1}, x_{n}\right) \leq d\left(x_{n-1}, x_{n}\right),
$$

yielding $b\left(d\left(x_{n-1}, x_{n}\right)\right) \leq b\left(d_{x_{n}}\right)$, thus, using the fact that $d_{x_{n}} \neq 0$,

$$
d_{x_{n}} \leq b\left(d_{x_{n}}\right) d\left(x_{n-1}, x_{n}\right) \leq \frac{d_{x_{n}}}{d\left(x_{n}, x_{n+1}\right)} d\left(x_{n-1}, x_{n}\right),
$$

and then

$$
d\left(x_{n}, x_{n+1}\right) \leq d\left(x_{n-1}, x_{n}\right) .
$$

By induction, either the process ends if $d\left(x_{k}, T\left(x_{k}\right)\right)=0$ for some $k \in \mathbb{N}^{*}$ or we obtain a sequence $\left(x_{n}\right)_{n \in \mathbb{N}} \subset D$ such that the sequences $\left(d\left(x_{n}, T\left(x_{n}\right)\right)\right)_{n \in \mathbb{N}} \subset \mathbb{R}$ and $\left(d\left(x_{n}, x_{n+1}\right)\right)_{n \in \mathbb{N}} \subset \mathbb{R}$ are decreasing. Denoting respectively by $d \geq 0$ and $s \geq d$ the limits of the decreasing sequences $\left(d\left(x_{n}, T\left(x_{n}\right)\right)\right)_{n \in \mathbb{N}}$ and $\left(d\left(x_{n}, x_{n+1}\right)\right)_{n \in \mathbb{N}}$, and assuming that $d>0$, we get, using (3.3), the contradiction

$$
d \leq \limsup _{t \downarrow s} c(t) d<d .
$$

It follows that $\lim _{n \rightarrow \infty} d\left(x_{n}, T\left(x_{n}\right)\right)=0$ and thus $\inf _{x \in D} d(x, T(x))=0$.

Here is our main result.

THEOREM 3.3. Let $(X, d)$ be a complete metric space and let $T \subset D \times X$ be a closed valued multifunction defined on a closed subset $D \subset X$. Assume that the function $x \mapsto d(x, T(x))$ is lower semicontinuous, and that there exist a function $\kappa:(0,+\infty) \rightarrow[0,1)$ and a nonincreasing function $b:(0,+\infty) \rightarrow[\underline{b}, 1]$, where $\underline{b} \in$ $(0,1]$, such that $\kappa(\cdot)<b(\cdot)$ and:

(1) T satisfies assumption $(\mathcal{H})$;

(2) $\quad \lim \sup _{t \downarrow s} b(t)^{-1} \kappa(t)<1$ for all $s \geq 0$.

Then, $\mathcal{F}_{T} \neq \emptyset$ and, if $\beta>0$ and $\delta>0$ satisfy $\sup _{t \in(0, \delta)} \kappa(t) b(t)^{-1} \leq 1-\beta$, then

$$
\underline{b} \beta d\left(x, \mathcal{F}_{T}\right) \leq d(x, T(x)) \quad \forall x \in D \text { such that } d(x, T(x))<\underline{b} \delta .
$$


Proof. Let us define $f: D \longrightarrow \mathbb{R}$ by $f(x)=d(x, T(x))$. From Lemma 3.2, we derive that $\inf _{x \in D} f(x)=0$ thus the set $[f<\underline{b} \delta]$ is nonempty. Given $x \in[0<f<\underline{b} \delta]$, we can find $z \in D \backslash\{x\}$ such that

$$
b(d(x, z)) d(x, z) \leq b\left(d_{x}\right) d(x, z) \leq d(x, T(x)),
$$

along with

$$
d(z, T(z)) \leq \kappa(d(x, z)) d(x, z)
$$

so that

$$
f(z)+(b(d(x, z))-\kappa(d(x, z))) d(x, z) \leq f(x),
$$

yielding

$$
f(z)+\left(1-\kappa(d(x, z)) b(d(x, z))^{-1}\right) b(d(x, z)) d(x, z) \leq f(x) .
$$

Now let $\beta>0$ and $\delta>0$ be such that $b(t)^{-1} \kappa(t) \leq 1-\beta$, for all $s \in(0, \delta)$. Then we get

$$
\underline{b} d(x, z) \leq b(d(x, z)) d(x, z) \leq b\left(d_{x}\right) d(x, z) \leq d(x, T(x))<\underline{b} \delta,
$$

so that $d(x, z)<\delta$ and then $f(z)+\underline{b} \beta d(x, z) \leq f(x)$ leading to the conclusion of the theorem by using Lemma 2.4 applied with $\mu=\underline{b} \delta$.

REMARK 3.4. In Theorem 3.3, the function $x \mapsto d(x, T(x))$ is required to be lower semicontinuous. This is the case if $T$ is Hausdorff upper semicontinuous, that is,

$$
\lim _{z \rightarrow x} e(T(z), T(x))=0
$$

for all $x \in X$. Indeed, we have $e(T(z), T(x)) \geq d(z, T(x))-d(z, T(z))$, so that

$$
\liminf _{z \rightarrow x} d(z, T(z)) \geq d(x, T(x)) .
$$

As a consequence of Theorem 3.3, we get an extension of the main result of [8] along three directions: the following corollary holds for nonself mappings, we use a nonconstant function $b(\cdot)$, and an estimate for the distance to the fixed-points set is available.

Corollary 3.5. Let $(X, d)$ be a complete metric space and let $T \subset D \times X$ be a closed valued multifunction defined on a closed subset $D \subset X$. Assume that the function $x \mapsto d(x, T(x))$ is lower semicontinuous, and that there exist a function $\kappa:(0,+\infty) \rightarrow[0,1)$ and a nonincreasing function $b:(0,+\infty) \rightarrow[\underline{b}, 1]$ where $\underline{b} \in(0,1]$, such that $\kappa(\cdot)<b(\cdot)$ and:

(1) $\left\{\begin{array}{l}\text { for all } x \in D \text { such that } d(x, T(x))>0, \text { there exists } z \in I_{b}^{x} \cap D \\ \text { such that } d(z, T(z)) \leq \kappa(d(x, z)) d(x, z) ;\end{array}\right.$

(2) $\quad \lim \sup _{t \downarrow s} b(t)^{-1} \kappa(t)<1$ for all $s \geq 0$. 
Then, $\mathcal{F}_{T} \neq \emptyset$ and, if $\beta>0$ and $\delta>0$ satisfy $\sup _{t \in(0, \delta)} \kappa(t) b(t)^{-1} \leq 1-\beta$, then

$$
\underline{b} \beta d\left(x, \mathcal{F}_{T}\right) \leq d(x, T(x)) \quad \forall x \in D \text { such that } d(x, T(x))<\underline{b} \delta .
$$

In the case where the functions $\kappa(\cdot)$ and $b(\cdot)$ are constant, it is possible to weaken assumption $(\mathcal{H})$ in order to get a generalization of the main result of [6].

THEOREM 3.6. Let $(X, d)$ be a complete metric space and let $T \subset D \times X$ be a closed valued multifunction defined on a closed subset $D \subset X$. Assume that the function $x \mapsto d(x, T(x))$ is lower semicontinuous, and that there exists $0 \leq \kappa<b$, such that

$$
\left\{\begin{array}{l}
\forall x \in D \text { such that } d(x, T(x))>0, \exists z \in D \backslash\{x\} \\
\text { such that } b d(x, z) \leq d(x, T(x)) \text { and } d(z, T(z)) \leq \kappa d(x, z) .
\end{array}\right.
$$

Then, $\mathcal{F}_{T} \neq \emptyset$ and

$$
(b-\kappa) d\left(x, \mathcal{F}_{T}\right) \leq d(x, T(x)) \quad \forall x \in D .
$$

Proof. Let us define $f: D \longrightarrow \mathbb{R}$ by $f(x)=d(x, T(x))$ and let $x \in[f>0]$, so that we can find $z \in D \backslash\{x\}$ such that $b d(x, z) \leq d(x, T(x))$ and $d(z, T(z)) \leq \kappa d(x, z)$, yielding $f(z)+(b-\kappa) d(x, z) \leq f(x)$, and then the conclusion of the theorem follows from Lemma 2.4 applied with $\mu=+\infty$.

REMARK 3.7. Observe that we do not require that $\kappa<1$ in Theorem 3.6. When applied to mappings, the previous theorem leads to a very light version of the classical Banach-Picard theorem: any continuous mapping $T: X \rightarrow X$ defined on a complete metric space for which we can find $0 \leq \kappa<b$ such that for all $x \in X$ with $T(x) \neq x$, there exists $z \in X$ satisfying $b d(x, z) \leq d(x, T(x))$ and $d(z, T(z)) \leq \kappa d(x, z)$ has a fixed point and $(b-\kappa) d\left(x, \mathcal{F}_{T}\right) \leq d(x, T(x))$ for all $x \in X$. A contraction $T$ : $X \rightarrow X$ of modulus $\kappa \in[0,1)$ satisfies the above assumption with $b=1$ and $z=T(x)$. The mapping $T: \mathbb{R}^{2} \rightarrow \mathbb{R}^{2}$ defined by $T=\left(\begin{array}{ll}\lambda & 0 \\ 0 & 1\end{array}\right)$ with $|\lambda|<1$ also satisfies the above assumption, but it is not a contraction. The fact that it is enough to require only $d(T(x), T(T(x))) \leq \kappa d(x, T(x))$ for all $x \in X$ instead of $d(T(x), T(z)) \leq \kappa d(x, z)$ for all $x, z \in X$ was implicit in [3].

\section{References}

[1] D. Azé, 'A survey on error bounds for lower semicontinuous functions', ESAIM Proc. 13 (2003), $1-17$.

[2] D. Azé and J.-N. Corvellec, 'A variational method in fixed point results with inwardness conditions', Proc. Amer. Math. Soc. 134(12) (2006), 3577-3583.

[3] J. B. Diaz and B. Margolis, 'A fixed point theorem of the alternative, for contractions on a generalized complete metric space', Bull. Amer. Math. Soc. 74 (1968), 305-309.

[4] I. Ekeland, 'On the variational principle', J. Math. Anal. Appl. 47 (1974), 324-353.

[5] - 'Nonconvex minimization problems', Bull. Amer. Math. Soc. 1 (1979), 443-474.

[6] Y. Feng and S. Liu, 'Fixed point theorems for multi-valued contractive mappings and multi-valued Caristi type mappings', J. Math. Anal. Appl. 317(1) (2006), 103-112. 
[7] A. Hamel, 'Remarks to an equivalent formulation of Ekeland's variational principle', Optimization 31(3) (1994), 233-238.

[8] D. Klim and D. Wardowski, 'Fixed point theorems for set-valued contractions in complete metric spaces', J. Math. Anal. Appl. 334(1) (2007), 132-139.

[9] N. Mizoguchi and W. Takahashi, 'Fixed point theorem for multivalued mappings on complete metric spaces', J. Math. Anal. Appl. 141 (1989), 177-188.

[10] S. B. Nadler, 'Multivalued contraction mappings', Pacific J. Math. 30 (1969), 475-488.

[11] J.-P. Penot, 'The drop theorem, the petal theorem and Ekeland's variational principle', Nonlinear Anal. 10 (1986), 813-822.

[12] S. Reich, 'Fixed points of contractive functions', Boll. Unione Mat. Ital. 5(4) (1972), $26-42$.

[13] P. V. Semenov, 'Fixed points of multivalued contractions', Funct. Anal. Appl. 36(2) (2002), 159-161.

[14] W. Takahashi, Existence theorems generalizing fixed point theorems for multivalued mappings, in Fixed Point Theory and Applications (Marseille, 1989), Pitman Res. Notes Math. Ser., 252, (Longman, Harlow, 1991) pp. 397-406.

[15] J. D. Weston, 'A characterization of metric completeness', Proc. Amer. Math. Soc. 64 (1977), 186-188.

\section{S. BENAHMED, ENSET D’Oran, BP 1523 El Ménaouer, 31000 Oran, Algérie e-mail: sfyabenahmed@yahoo.fr}

D. AZÉ, Institut de Mathématiques de Toulouse UMR CNRS 5219, Université Paul Sabatier, 118 Route de Narbonne, 31062 Toulouse cedex 4, France e-mail: aze@mip.ups-tlse.fr 